\title{
Instrumental Activities Domain
}

National Cancer Institute

\section{Source}

National Cancer Institute. Instrumental Activities Domain. NCI Thesaurus. Code C129987.

A domain of the Activity Card Sort (ACS) related to crucial activities that may be part of an individual's routine such as driving, paying bills, and child care. 